\title{
Bypassing the bottleneck: intentional hepatitis C transmission with organ transplant
}

\author{
Christine M. Durand and Michael A. Chattergoon \\ Department of Medicine, Johns Hopkins University School of Medicine, Baltimore, Maryland, USA.
}

\begin{abstract}
Solid organ transplantation from hepatitis C virus-positive (HCV-positive) deceased donors into HCV-negative recipients is a recent approach aimed to expand the donor organ pool in the setting of severe shortage. Good shortterm outcomes have been reported with this approach in combination with direct-acting antivirals. In this issue of the $J C I$, Zahid and colleagues have characterized early viral kinetics and the genetic landscape of donor-torecipient $\mathrm{HCV}$ transmission using single-genome sequencing. In seven HCVnegative recipients of four HCV-positive donor organs, productive infection with a highly diverse viral population was seen by day three after transplant. The degree of genetic diversity seen in recipients of HCV-positive organs was unlike the narrow genetic bottleneck typically observed with acute $\mathrm{HCV}$ acquisition from intravenous drug use or sexual activity. All recipients achieved HCV cure with treatment. The consequences of acute infection with a genetically diverse HCV population are unknown; however, early clinical experience with this transplantation strategy is promising.
\end{abstract}

transplantation and treated upon detection of HCV viremia with a 12-week course of grazoprevir/elbasvir (GZR/EBR), a so-called transmit and treat approach. Universal donor-to-recipient HCV transmission was detected by day three. All recipients $(n=29)$ achieved HCV cure except for a single heart recipient, who died from acute antibody-mediated cardiac allograft rejection thought to be unrelated to $\mathrm{HCV}$.

In this issue, Zahid and colleagues present an in-depth virologic analysis of seven cases (six kidney recipients and one heart recipient) of de novo HCV infection acquired from organs of four HCV-positive donors within these trials (11). The authors used single genome sequencing (SGS) to characterize the viral kinetics and $\mathrm{HCV}$ genetic population diversity in the donor at the time of organ recovery and in the recipient on day three, prior to initiation of DAAs. This method of limiting-dilution PCR, though labor intensive, allows for reliable identification of unique viral variants, thereby reducing the potential for amplifying PCR-induced errors or in vitro recombination events.

Zahid et al. first observed that $\mathrm{HCV}$ viral RNA increased rapidly in all recipients prior to treatment initiation on day three, with peak viral loads ranging from 2.9-5.3 $\log _{10} \mathrm{IU} / \mathrm{mL}$ (11). This exponential increase indicates that virus detected in the recipient was not merely the result of transfer from the donor organ but represented productive de novo infection of the recipient liver. Of note, in the single heart transplant recipient studied, the peak viral load was one log lower than the peak viral load in the kidney recipient of the same donor.

Second, Zahid and colleagues observed that by day three, recipients harbored many distinct viruses, representing nearly all HCV lineages identified in donor plasma at the time of organ procurement. Maximum likelihood phylogenetic trees demonstrated interspersion of donor and recipient viruses - except in the case of one donor, who harbored a variant carrying a large 
A

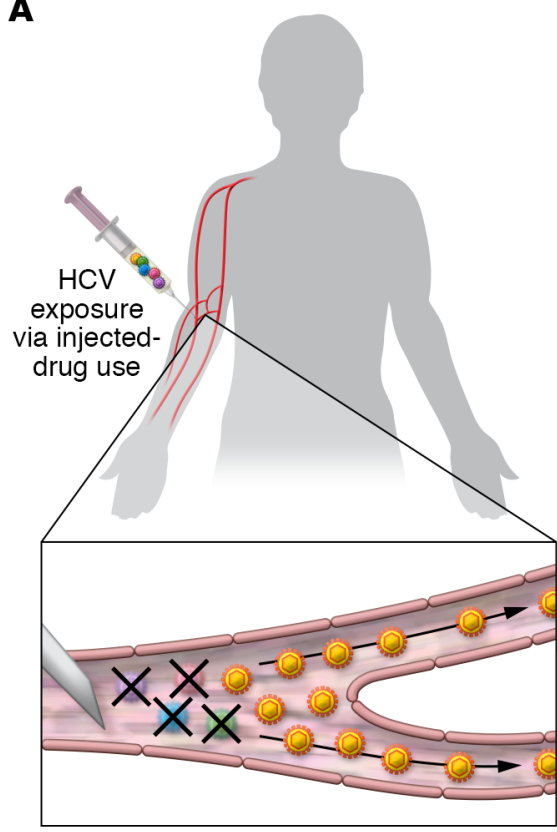

deletion with unknown fitness in vivo. Population relatedness, which is measured with a statistical test known as a genealogical sorting index (GSI), demonstrated that the viral population diversity in the chronically infected donors and acutely infected recipients were indistinguishable. In contrast, acute HCV, acquired parenterally or sexually, typically exhibits a stringent population bottleneck (Figure 1A). This occurs because infection usually results from the acquisition of a very limited number of viruses (called transmitted founder variants) that expand exponentially and with essentially random early mutational events (12). In contrast, Zahid and colleagues observed minimal genetic bottleneck, with innumerable transmitted founder viruses, likely due to a much larger inoculum of virus carried by the donor organ (Figure 1B) (11). This pattern was seen in transmission cases from donors with high $\left(7.3 \log _{10} \mathrm{IU} / \mathrm{mL}\right)$, intermediate $\left(5.2 \log _{10} \mathrm{IU} / \mathrm{mL}\right)$, or unknown viral loads, and was present in both kidney and heart recipients. Interestingly, the authors also provided evidence that even heavily deleted HCV clones may transmit in the absence of a stringent bottleneck, providing strong evidence for transcomplementation.

\section{Considerations and future directions}

What are the implications of a large viral inoculum and permissive genetic bottleneck with HCV transmission in organ

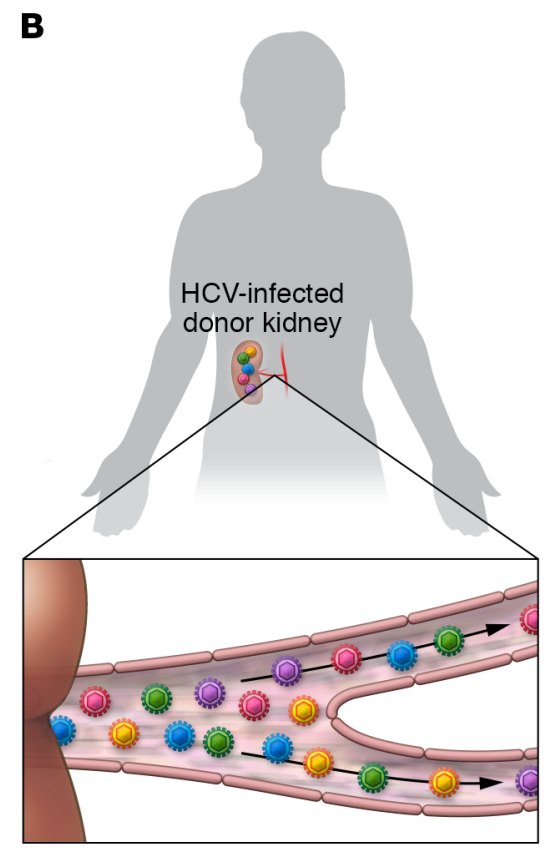

Figure 1. Transmission of HCV from donor organs does not encounter the genetic bottleneck observed in other routes of HCV transmission. (A) Sexual and parenteral transmission of HCV, such as occurs with i.v. drug use, results in a stringent bottleneck in which a single strain expands and dominates at the acute phase of infection. (B) In this issue of the $J C$, Zahid and colleagues demonstrate that this bottleneck is lacking in HCV infection acquired via receipt of organs from HCV-positive donors. Moreover, the viral diversity observed in organ recipients during acute infection mimics the viral diversity of the donor.

transplantation? One theoretical concern is that productive infection by a multiplicity of viruses may lead to treatment failure. Early studies with IFN treatment showed acute HCV infection was more likely to be cured than chronic HCV infection (13), suggesting that viral populations with low genetic diversity might be more susceptible to clearance. However, with potent DAAs, viral diversity may not correlate with treatment success. Another concern is that high permissiveness might result in infection with DAA-resistant viruses, thereby jeopardizing treatment success. The current study by Zahid et al. focuses on the $5^{\prime}$ half of the HCV genome and thus does not capture relevant substitutions associated with resistance to NS5A and NS5B inhibitors (11). Regardless, no treatment failures have been reported in the single center trials $(9,10$, $14,15)$ and observational studies $(16,17)$ of $\mathrm{HCV} \mathrm{D}^{+} / \mathrm{R}^{-}$transplantation to date. These studies include kidney, liver, heart, and lung transplants and different DAA regimens. However, the overall number of published $\mathrm{HCV} \mathrm{D}^{+} / \mathrm{R}^{-}$transplant cases remain small. In USHER, there is a reference to one $\mathrm{HCV} \mathrm{D}^{+} / \mathrm{R}^{-}$kidney nonresponder who had HCV breakthrough at day 20 and required intensification of DAA therapy, which included the addition of sofosbuvir and ribavirin to GZR/ EBR and extension of treatment from 12 to 16 weeks. In THINKER, 3 of 20 (15\%)
$\mathrm{HCV} \mathrm{D}^{+} / \mathrm{R}^{-}$recipients were infected with NS5A-resistant viruses. In all of these cases, ribavirin was added and cure achieved.

Could transmission of a large, genetically diverse viral population lead to treatment failure and would there be ways to mitigate this? Perhaps. Several DAA strategies have been used successfully with $\mathrm{HCV} \mathrm{D}^{+} / \mathrm{R}^{-}$transplantation. In transmit and treat, recipients are monitored after transplantation and treated after viremia is detected. THINKER and USHER used this approach, initiating treatment within days of viremia detection. Within observational studies $(16,17)$, providers had to wait for insurance approval to administer DAAs, sometimes delaying treatment for several months. Alternatively, DAAs can be used as prophylaxis. The first trial to explore prophylactic DAAs was EXPANDER (Exploring Renal Transplants Using Hepatitis C Infected Donors for HCV-negative Recipients), in which ten $\mathrm{HCV} \mathrm{D}^{+} / \mathrm{R}^{-}$kidney recipients received one preoperative dose of GZR/EBR followed by a 12-week treatment course (14). In EXPANDER, no recipients had exponential increases of HCV viremia, an indicator of productive infection. In DONATE HCV, a recent heart and lung transplant trial, investigators provided four weeks of sofosbuvir/velpatasvir as postexposure prophylaxis for all $36 \mathrm{HCV}$ $\mathrm{D}^{+} / \mathrm{R}^{-}$lung and eight heart recipients (15). There is no consensus on whether prophylaxis versus transmit and treat is preferred. 
Potential benefits of prevention include avoiding HCV-related complications, such as fibrosing cholestatic hepatitis, which can be fatal in immunosuppressed patients (18). Also, a shorter DAA prophylactic course - such as that used in the DONATE HCV trial - would mitigate the higher costs of this strategy, which requires treating all recipients. On the other hand, no parenteral DAA regimen is available, and the transmit and treat approach allows physicians to wait until recipients are stable and can take oral medications after transplantation. Since levels of DAAs can be affected by crushing, reliable delivery of DAAs via nasogastric tubes is uncertain (19). If the diverse viral population afforded by a permissive genetic bottleneck does impact treatment response, prophylaxis might be more efficacious by blocking establishment of infection.

Of interest, a novel method of inactivating $\mathrm{HCV}$ using germicidal light-based therapies during ex vivo donor lung perfusion has been used in an animal model to prevent HCV transmission during transplantation (20). Early results suggest this technique does not eliminate transmission but it does reduce HCV RNA levels in the organ by more than $90 \%$. This approach might narrow the genetic bottleneck by eliminating most of the donor virus, though a $90 \%$ reduction in HCV RNA levels can be observed in a matter of hours of DAA treatment.

In summary, Zahid and colleagues describe the distinctive virologic landscape of $\mathrm{HCV}$ transmission during solid organ transplantation. Unlike injection drug use, solid organ transplant does not impose a restrictive bottleneck on the donor's viruses, allowing transmission of multiple HCV clones with the potential for transmission of clones harboring DAAresistant variants. The practice of $\mathrm{HCV}$ $\mathrm{D}^{+} / \mathrm{R}^{-}$transplantation will undoubtedly continue to expand given the urgent need to optimize the use of donated organs. Further studies to better understand the clinical consequences of this unique mode of $\mathrm{HCV}$ acquisition are essential.

Address correspondence to: Christine M. Durand, Johns Hopkins University School of Medicine, 725 North Wolfe Street, Suite 211, Baltimore, Maryland 21205, USA. Phone: 410.955.5684; Email: christinedurand@ jhmi.edu.

1. UNOS Transplant Trends. https://unos.org/ data/transplant-trends. Accessed May 17, 2019.

2. Scholl L, Seth P, Kariisa M, Wilson N, Baldwin G. Drug and opioid-involved overdose deaths United States, 2013-2017. MMWR Morb Mortal Wkly Rep. 2018;67(5152):1419-1427.

3. Durand CM, et al. The drug overdose epidemic and deceased-donor transplantation in the United States: a national registry study. Ann Intern Med. 2018;168(10):702-711.

4. Bowring MG, et al. Changes in utilization and discard of HCV antibody-positive deceased donor kidneys in the era of direct-acting antiviral therapy. Transplantation. 2018;102(12):2088-2095.

5. Bowring MG, et al. Changes in utilization and discard of hepatitis $\mathrm{C}$-infected donor livers in the recent era. Am J Transplant. 2017;17(2):519-527.

6. Reau N, et al. Glecaprevir/pibrentasvir treatment in liver or kidney transplant patients with hepatitis $\mathrm{C}$ virus infection. Hepatology. 2018;68(4):1298-1307.

7. Colombo M, et al. Treatment With ledipasvir-sofosbuvir for 12 or 24 weeks in kidney transplant recipients with chronic hepatitis $C$ virus genotype 1 or 4 infection: a randomized trial. Ann Intern Med. 2017;166(2):109-117.

8. Reese PP, Abt PL, Blumberg EA, Goldberg DS. Transplanting hepatitis C-positive kidneys.
NEngl J Med. 2015;373(4):303-305.

9. Reese PP, et al. Twelve-month outcomes after transplant of hepatitis $\mathrm{C}$-infected kidneys into uninfected recipients: a single-group trial. Ann Intern Med. 2018;169(5):273-281.

10. McLean RC, et al. Transplanting hepatitis C virus-infected hearts into uninfected recipients: a single-arm trial [published online ahead of print February 15, 2019]. Am J Transplant. https://doi.org/10.1111/ajt.15311.

11. Zahid KJ, et al. High multiplicity infection following transplantation of hepatitis $\mathrm{C}$ virus-positive organs. J Clin Invest. 2019;129(8):3134-3139.

12. Abayasingam $A$, et al. Genomic characterization of hepatitis $C$ virus transmitted founder variants with deep sequencing. Infect Genet Evol. 2019;71:36-41.

13. Jaeckel E, et al. Treatment of acute hepatitis $C$ with interferon alfa-2b. N Engl JMed. 2001;345(20):1452-1457.

14. Durand CM, et al. Direct-acting antiviral prophylaxis in kidney transplantation from hepatitis $\mathrm{C}$ virus-infected donors to noninfected recipients: an open-label nonrandomized trial. Ann Intern Med. 2018;168(8):533-540.

15. Woolley AE, et al. Heart and lung transplants from HCV-infected donors to uninfected recipients. N Engl JMed. 2019;380(17):1606-1617.

16. Schlendorf $\mathrm{KH}$, et al. Early outcomes using hepatitis $\mathrm{C}$-positive donors for cardiac transplantation in the era of effective direct-acting anti-viral therapies. J Heart Lung Transplant. 2018;37(6):763-769.

17. Kwong AJ, et al. Liver transplantation for hepatitis $\mathrm{C}$ virus (HCV) non-viremic recipients with $\mathrm{HCV}$ viremic donors. Am J Transplant. 2019;19(5):1380-1387.

18. Pellicelli AM, et al. Sofosbuvir plus daclatasvir for post-transplant recurrent hepatitis C: potent antiviral activity but no clinical benefit if treatment is given late. Dig Liver Dis. 2014;46(10):923-927.

19. Oberoi RK, Zhao W, Sidhu DS, Viani RM, Trinh R, Liu W. A phase 1 study to evaluate the effect of crushing, cutting into half, or grinding of glecapre$\mathrm{vir} /$ pibrentasvir tablets on exposures in healthy subjects. JPharm Sci. 2018;107(6):1724-1730.

20. Galasso $\mathrm{M}$, et al. Inactivating hepatitis $\mathrm{C}$ virus in donor lungs using light therapies during normothermic ex vivo lung perfusion. Nat Commun. 2019;10(1):481. 